Institute for preparing for us the experimental material and for biochemical data.

$\mathrm{X}$-ray examination of other nucleic acids and polynucleotides is in progress.

W. T. Astburx.

Florence O. Bell.

Textile Physics Laboratory,

University of Leeds.

March 25.

${ }^{1}$ Cf. Schmidt, W. J., "Die Doppelbrechung von Karyoplasma, Zytoplasma und Metaplasma" (Berlin, 1937).

${ }^{2}$ Signer, R., Caspersson, T., and Hammarsten, E., NATURE, 141, 122 (1938).

4f. Astbury, W. T., NATURE, 140, 968 (1937).

${ }^{5}$ Cox, E. G., NATURE, 130, 205 (1932). Cox, E. G., and Goodwin, T. H., J. Chem. Soc., 769 (1936).

\section{Structure of Metals}

Is the note by Dr. A. Müller ${ }^{1}$ with the above title, the scatter of spots in high-dispersion X-ray photographs was interpreted as indicating variations in lattice constant of individual crystals of the order of $1 / 1000$ to $1 / 2000$.

Prof. W. L. Bragg and H. Lipson ${ }^{2}$ afterwards advanced the tentative explanation of the effects observed by Müller without making the deduction that the lattice constant varies. The scatter of spots in stationary photographs deduced from the effect shown by Prof. Bragg and Lipson is equivalent to a $\Delta d / d$ value of $1 / 2000$ approximately, for $l$ equal to $10^{-4} \mathrm{~cm}$.

There are two further effects which can give rise to scatter of spots from grains of identical lattice constant. (1) A purely geometrical effect due to the finite convergency of the X-ray beam. (2) The fact that the $K \alpha$ lines have a finite width. The first effect, the purely geometrical effect, can give rise to an angular scatter of spots which for a given constant diameter of pinhole or diaphragm becomes less as the specimen to film distance increases, provided a focusing technique is utilized; for example, pinhole, diffraction line and specimen surface lie on the circumference of the same circle.

If a reference sphere is drawn, it is easily seen that a reflection is possible provided the normal to the set of planes giving the reflection lies within a band which is determined by the maximum angle of convergence of the incident X-ray beam subtended at the specimen surface. Furthermore, the reflection spots themselves can be within a band of similar angular width. The angular width of this band, $\Delta \omega$, or what is the same thing, the possible scatter of the reflection spots from crystals of identical lattice constant, is given by

$$
\Delta \omega=\frac{\text { diameter of pinhole }}{\text { specimen-diaphragm distance. }}
$$

Such a scatter might erroneously be taken as arising from a variation in lattice constant which for normal cameras of $5-10 \mathrm{~cm}$. film-specimen distance can amount to 1 part in 1000 to 1 part in 2000 .

Dr. Müller has kindly informed me that his high dispersion photographs were taken at $30 \mathrm{~cm}$. with a $0 \cdot 3 \mathrm{~mm}$. pinhole. The purely geometrical scatter accounts only for a variation $\Delta d / d=1 / 20,000$ approximately and does not explain Müller's result.

The second effect, the more important effect, is that due to the finite width of the $K \alpha$ lines usually used; it does not depend on the geometry of the camera system, though it must be considered in conjunction with the geometric effect in determining the conditions in which $\alpha_{1}$ and $\alpha_{2}$ reflections may intermingle.

Values for the half value width $\Delta \lambda$ of the $K$ lines of copper, iron and cobalt taken from Compton and Allison ${ }^{3}$ are as follows :

Full width at half max. of $K \alpha$ lines.

\begin{tabular}{|c|c|c|c|c|}
\hline Element & Line & $\begin{array}{c}\text { Wave-length } \\
\text { in X.U. }\end{array}$ & $\Delta \lambda$ in X.U. & $\Delta \lambda / \lambda$ \\
\hline Iron & $K \alpha_{2}$ & 1936 & 1.18 & $1 / 2000$ approx. \\
& $K a_{1}$ & 1932 & 1.02 & $"$ \\
Cobalt & $K a_{2}$ & 1789 & 0.95 &, \\
& $K a_{1}$ & 1785 & 0.82 &, \\
Copper & $K \alpha_{2}$ & 1541 & 0.77 &, \\
& $K \alpha_{1}$ & 1537 & 0.58 &, \\
\hline
\end{tabular}

This definite half-value width would account for variations in angular scatter of spots from crystals of identical lattice spacing equivalent to $\Delta d / d=1 / 2000$ as an upper limit, whilst since the half-value width is purely an arbitrary method of estimating line width, spots could occur possibly even down to $\triangle d / d=$ $1 / 1000$.

It is of special importance to take these effects into account when using rotating or oscillating film back reflection methods of determining lattice constants, as in internal stress measurements; that is, one must average over a large number of spots, otherwise serious errors in lattice constant and in internal stresses deduced will result.

X-Ray Service Laboratories,

R. A. Stephen.

Philips Lamps, Ltd., London.

Mar. 21.

1 NatURE, 140, 1012 (Dec. 11, 1937).

2 NATURE, 141, 367 (Feb. 26, 1938).

" "X-Rays in Theory and Experiment", by Compton and Allison.

\section{Biological Synthesis of Amino Acids from Atmospheric Nitrogen}

Sумвготіс nitrogen fixation in leguminous root nodules proceeds, according to our conception, in the following manner :

$\mathrm{N}_{2} \rightarrow$ hydroxylamine.

$\rightarrow$ oxime of oxalacetic acid $\rightarrow l$-aspartic in plant

This conception is based, in the first place, on the investigation of excreted nitrogen compounds from the root nodules. In extreme cases up to 80 per cent of the total fixed nitrogen is excreted into the medium from the root nodules during the growth of the pea. This nitrogen is, as regards a small part, oximenitrogen (usually 1-2 per cent of the total nitrogen) but mainly amino nitrogen, belonging to $l$-aspartic acid and to its decomposition product $\beta$-alanine (not to lysine, as we first assumed). As the legume bacteria decarboxylate $l$-aspartic acid forming $\beta$ alanine, $l$-aspartic acid is the first amino acid excreted $^{1}$. Its excretion from young nodules in large amounts proves that it is formed in nitrogen fixation as a primary amino acid, from which other amino acids are then formed.

In investigating the further transformation of $l$-aspartic acid in the host plant, it has been noted that if $l$-aspartic acid and, for example, pyruvic acid, are added to the mass of crushed pea plants, the amino 